UCRL-JC-105586

PREPRINT

$$
\text { hennived by } 087
$$

AUG 28 1991]

\title{
Transport and Error Sensitivity in a Heavy-Ion Recirculator
}

\author{
W.M. Sharp, J.J. Barnard, and S.S. Yu
}

This paper was prepared for submittal to the 1991 IEEE Particle Accelerator Conference May 6-9, 1991, San Francisco, CA

May 1991

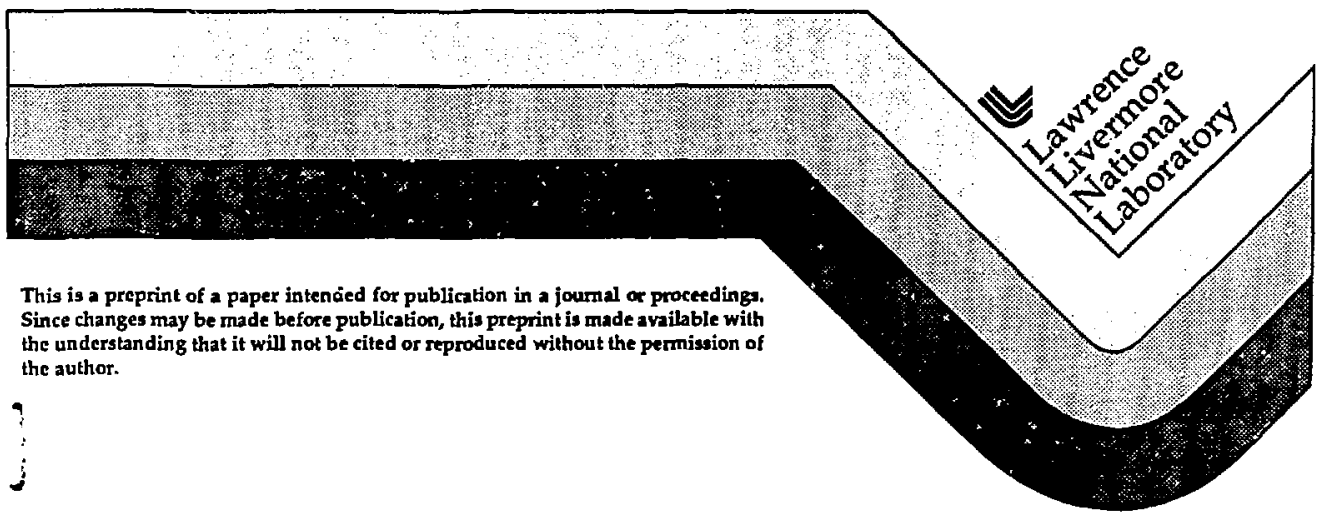

This is a preprint of a paper intencied for publication in a journal or proceedings. Since changes may be made before publication, this preprint is made available with the understanding that it will not be cited or reproduced without the permission of the author.

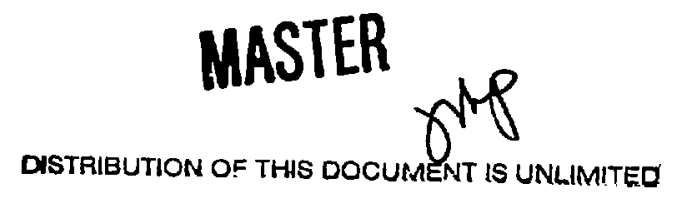




\section{DISCLIEAER}

This document was prepared as an account of work sponsnred by an askency of the United States Cuvernment. Neither the United Sitats Covernment nor the University of Calitornia nor any al their emplojecx, makes any marraniy; express or implind or assumes eay legal lisbility or essponsibility for the eccuracy, complet eness. ar usefulnest of any information apparalus product, of prectess disclosed, of represents that its use nould not inftinge

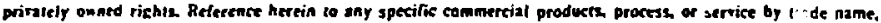
trademark, manufacturet. or osherwise, does aot netessarily conslitute or imply irs endorxemeat, recomme "ition, or iarorine by the United Slates Government or the University of California. The views and opinions authors expressed berein do ner necessarily state or refect these of ithe United States Corernment or the Uni. isity of

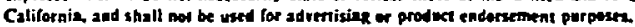

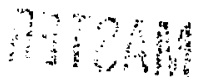




\title{
Transport and Error Sensitivity in a Heavy-Ion Recirculator ${ }^{* *}$
}

\author{
W. M. Sharp, J. J. Barnard, and S. S. Yu \\ Lawrence Livermore National Laboratory, University of California \\ Liyermore, California 94550
}

\section{Abstract}

An envelope code has been developed to facilitate the design of a recirculating accelerator for a heavy-ion fusion reactor. A noyel feature of the model is the treatment of the beam charge density as a Lagrangian fluid in the axial direction. Transport results for a preliminary recirculator design are presented, and sensitivity of the transport to errors in the magnet strength is discussed.

\section{Introduction}

A fast-running envelope code CIRCE has been developed to carry out the detailed lattice design for the recirculating heavy-ion accelerator[1] proposed by Lawrence Livermore National Laboratory (LLNL). The model combines an envelope description of the beam transverse dynamics with a fluid-like treatment of axial dynamics. Appropriate terms are included to account for the effects of image forces, beam emittance, and space-charge in the limit of paraxial motion, and the heam is focused and accelereted by a user-specified lattice of time-varying bend magnets, quadrupoles, and accelerating modules. Since the code has previously been described in detail[2], we only review the salient features of the model here. The main part of the paper summarizes code results inr a preliminary lattice design, looking in particular at the effects of lattice transitions and magnet errors.

\section{Model}

As discussed in Ref. [2], the beam transverse dynamics is modeled in CIRCE by a set of envelope equations similar to that formulated first by Kapchinskij and Vladimirskij and later adapted by Lee, Close, and Smith[3]. The beam transverse distribution function is assumed to be uniform and elliptical in each phase-space plane, and in the absence of an axial magnetic field the coordinate-space ellipse may be taken to be unskewed. The treatment is first orcier in the ratio of the beam radii and centroid displacements to the beam-pipe radius, making it appropriate to represent bend magnets and quadrupoles by idealized expressions and to neglect higher-order multipole fields. In the present version of CIRCE, only single-function magnets are used, and axial fringe fields are neglected. Also, we assume a circular beam pipe of infinite conductivity and radius $R$. With these assumptions, equations for the centroid displacement in the plane of the accelerator $X$ and $t$ be coordinate-space radii $a$ and $b$ are derived in the conventional manner.

* Work petformed under the auspices of the U. S. Department of Energy by Lawrence Livermore National Laboratory under contract W-7405ENG-48.
Axial dynamics is modeled by treating slices of the beam as Lagrangian fluid elements characterized by an axial velocity $\beta c$ and the time $\tau$ that the slice arrives at an axial location $s$. This approach implicitly assumes that the beam has a negligible longitudinal temperature and that the slices remain approximately collinear. If we assume that slice boundaries remain perpendicular to the beam-pipe axis, then the equation for $\tau$ is found from orbit kinematics to be

$$
\frac{d \tau}{d s}=\frac{1}{\beta c}\left(1+\frac{X}{\rho}\right)
$$

where $\rho$ is the local beam-pipe radius and we have assumed paraxial motion. An approximate $\beta$ equation is obtained by retaining only the electrostatic force in the single-particle motion equations and averaging the axial component over the beam elliptical cross-section. For a beam with an ion mass $M$ and charge state $q$, we obtain

$$
\frac{d \beta}{d s}=\frac{q e}{\beta M c^{2}}\left(1+\frac{X}{\rho}\right)\left(E_{e x t}+E_{s c}\right) .
$$

Here, the average external electric field $E_{\text {ext }}$ is approximated by the voltage across accelerating modules divided by the gap length, and the space-charge field is approximated by

$E_{s c} \approx \frac{1}{4 \pi \epsilon_{0}}\left[\ln \left(\frac{2 R^{2}}{a^{2}+b^{2}}\right)+\frac{1}{2}\right]\left[\frac{\partial}{\partial \tau}\left(\frac{I_{b}}{\beta^{2} c^{2}}\right)+\frac{I_{b}}{\beta^{2} c} \frac{d \beta}{d s}\right]$,

where $I_{b}$ is the beam current and $R$ is the beam-pipe radius In deriving the space-charge field, the radial electrostatic field is assumed to vary over a much shorter scale length than $E_{s c}$, and the continuity equation is used to convert derivatives with respect to $s$ into $\tau$ derivatives. Combining Eqs. (2) and (3), we obtain an equation for $d \beta / d s$ in ternus of $E_{e x t}$ and the time derivative of $I_{b} / \beta^{2}$.

One important omission from the CIRCE model is the absence of phase-mix damping in either the longitudinal or transverse directions. Because of this shortcoming, spacecharge waves and betatron motion persist indefinitely, rather than contributing, as they should, to growth of the longitudinal or transverse emittance. There is also no provision for emittance growtl due to scattering of the background gas.

\section{Results}

Design work with CIRCE has concentrated on model. ing transport in and extraction from the final rin $r$ of the four-ring LLNL recirculator and studying the effects of lattice non-uniformity and magnet errors. The approach used here is first to model a uniform circular lattice with none of 

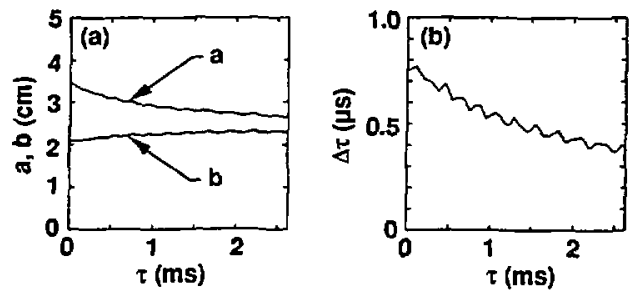

Fig. 1 Envelope parameters for a parabolic beam during fifty laps around the idealized recirculator ring: (a) beam radii $a$ and $b$ before an $x$-focusing quadrupole; (b) beam duration $\Delta \tau$.

the complication found in a realistic design. Thn straight section required for injection and extraction as well as the lattice distortions needed for matching smoothly into and out of the straight section are modeled separately, and the effects of magnet errors and initial mismatches are studied by making appropriate changes in the basic circular lattice or the initjal beam configuration.

The lattice of the basic circular ring consists of alternating $2.0 \mathrm{~T}$ focusing and defocusing superconducting quadrupoles with a half-lattice period $L$ of $2.865 \mathrm{~m}$ and an occupancy of 0.35 . Between each pair of quadrupoles is a $1.5 \mathrm{~m}$ sector bend magnet flanked by a pair of accelerating gaps. The entire ring consists of 592 identical lattice periods and is $3392 \mathrm{~m}$ long. The bend field $B_{y}$ is assumed in the present simulations to increase linearly with time $t$, corresponding to a constant beam-energy gain per unit length. We use two accelerating gaps per quadrupole because this arrangement reduces the gap voltage and allows a better approximation to a linear energy gain than a one-gap configuration. At injection, the ion beam has an energy of $1 \mathrm{GeV}$, a duration $\Delta \tau$ of $750 \mathrm{~ns}$, and a normalized emittance of $10^{-5} \mathrm{~m}$-rad. The initial beam velocity is assumed to be uniform, and the current is taken to vary parabolically along the length of the beam, with a peak value of $132 \mathrm{~A}$.

\section{A. Transport}

For the nominal accelerating gradient, the beam energy inereases from $1 \mathrm{GeV}$ to $10 \mathrm{GeV}$ in fifty laps around the last recirculator ring. Since the initial phase advance per lattice period $\sigma_{0}$ is about $80^{\circ}$ and each beam slice is initially matched, motion of the beam envelope is expected to be stable and free of betatron oscillations, and this regularity is seen in CIRCE runs. The beam radii and centroid are found to oscillate on the scale of a lattice period, and the plots in Fig. 1 show a vary gradual change in the beam length and average radius due the the changing beam velocity. In the radius plots of Fig. $1 a_{1} a$ is systematically larger than $b$ because the average values plotted are obtained at the beginning of a quadrupole that focuses in the $x$-plane. Some fluctuation is seen in the $\Delta r$ plot because the $E_{z}$ ramp is calculated using the initial densityweighted average values of $a_{3} b$, and $\beta_{1}$ whereas the beam

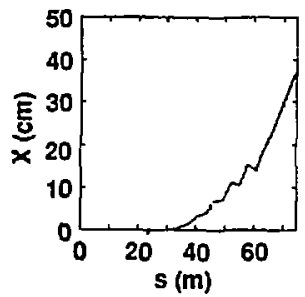

Fig. 2 Centroid location in extraction section for several beam slices with velocity errors between $\pm 1 \%$

space-charge field depends on the local time-dependent values during transport.

\section{B. Extraction}

Extraction from the final recirculator ring is complicated by the high magnetic rigidity of a $10 \mathrm{GeV}$ heavy-ion beam. The method planned for the LLNL recirculator is to bend the beam away from the axis over several lattice periods in a large-aperture rectangular beam pipe. The current design uses eleven $1.5 \mathrm{~m}$ bend magnets each with a strength of $0.34 \mathrm{~T}$. The relatively weak bend-field strength allows the magnets to be turned on between successive beam passes, and the odd number guarantees that the last bend be followed by a defocusing magnet. Focusing in the extraction section is provided by superconducting rectangular quadrupoles similar to the type developed by Hand and Panofsky[4], and to minimize perturbations to the orbit of an undeflected beam, the quadrupole lattice has the same period, occupancy, and transverse gradient as the main lattice.

Although CIRCE at present has no provision for modeling rectangular quadrupoles, the magnets are indistinguishable in the linear approximation from large-radius circular quadrupoles. We therefore model the extraction section by an appropriate lattice of circular quadrupoles, sector bends, and drifts with a radius of $0.244 \mathrm{~m}$, with several lattice periods of the nominal $0.61 \mathrm{~m}$ radius before and after it. This lattice is found in CIRCE simulations to give $15 \mathrm{~cm}$ centroid deflection with an extraction angle of $10^{\circ}$. Although the number of lattice periods in the extraction section was chosen to minimize the spatial dispersion of the extracted beam, the extraction angle is also found to be insensitive to errors in beam energy. Centroid plots for a serjes of beam slices with $\beta$-values ranging between $\pm 1 \%$ of the design value are superimposed in Fig. 2. The fact that the curves are nearly indistinguishable indicates that the chromaticity of the extraction lattice is quite low.

\section{Effect of Non-Circular Lattice}

The extraction section shouid be straight in order to simplify the bend-magnet design, and the lattice must be distorted to match smoothly into this section. This matcliing is done by making the main lattice nearly semicircular and joining it tangentially to the straight section by a pair 

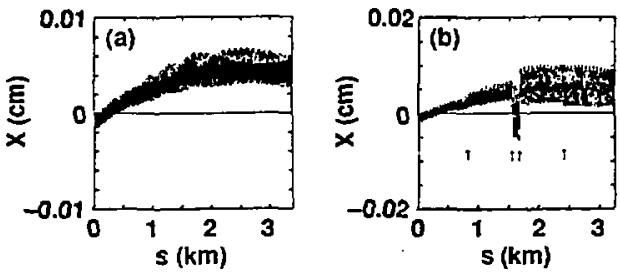

Fig. 3 Centroid histories as a function of s: (a) circular lattice; (b) near-circular lattice with straight extraction section

of quarter-circle arcs of somewhat smaller radius. Simulations of this lattice show that the changes in bend radii between the main lattice and the matching arcs and between the matching arcs and the straight section cause abrupt changes in the centroid motion unless either $X=0$ or the beam moment um per unit charge exactly equals $B_{y} \rho$. The effect of these transitions is seen by comparing the centroid histories in Fig. 3. The centroid for a slice at the beam midpoint in the nominal circular lattice, shown in Fig. 3a, increases gradually with propagation distance $s$, whereas the plot in Fig. 3 b for a similar case with matching arcs and a straight section shows abrupt changes in amplitude and median value of lattice oscillations at each transition point, marked by arrows. The largest changes occur at the entry and exit of the straight section, due to the sudden change in $B_{y}$ and $\rho$ there. The increases in the amplitude of centroid oscillations are found to be cumulative from lap to lap and, if phase mixing were in the model, would lead to unacceptable emittance growth.

Although simulation indicates that sudden changes in the lattice bend radius introduce serious mismatches, the increase in centroid oscillations is substantially reduced by changing the radius in steps over several lattice periods. It is also helpful to have the beam near the beam-pipe axis at all transitions in the lattice. To accomplish this, the bend-field ramp in each magnet must be timed to match the beam momentum there, and time-dependent steering is probably needed before transition points to further reduce the residual centroid displacement.

\section{Effect of Current Profile}

The parabolic current profile used in the basic case is impractical because the linearly varying $E_{z}$ field, needed to balance longitudinal space charge, allows the beam to shrink excessively during acceleration. If the $E_{\text {ext }}$ profile is shaped by pulse-forming networks, then the acceleratingpulse duration cannot be varied from lap to lap, so any beam shrinkage degrades the accelerator efficiency.

To balance the space charge in flat-topped beam, we need a non-linear time variation in $E_{t z y}$. If we neglent variations of $a, b$, and $\beta$ in $\mathrm{Eq}$. (3), we find from $\mathrm{Eq}$. (2)
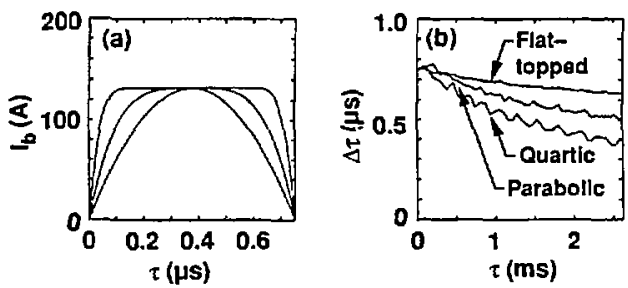

Fig. 4 Comparison of beams with different profles during fifty laps around the idealized recirculator ring:

(a) initial current profiles; (b) beam durations $\Delta r$.

that the appropriate time-varying part of $E_{e x t}$ is

$$
\Delta E_{\mathrm{ext}} \approx-\frac{1}{4 \pi \epsilon_{\mathrm{o}}}\left[\ln \left(\frac{2 R^{2}}{\bar{a}^{2}+\bar{b}^{2}}\right)+\frac{1}{2}\right] \frac{L}{L_{\mathrm{gap}}} \frac{1}{\beta^{2} c^{2}} \frac{\partial I_{b}}{\partial \tau} \text {, }
$$

where $\bar{a}$ and $\bar{b}$ are the matched beam radii averaged over a lattice period. The model beam density profile used here is flat in the the central part of the beam but falls to zero at the ends like $\tau^{4}$. A quartic variation is chosen because the corresponding $\Delta E_{e x i}$ is smooth and because the large electric-field gradient at the beam ends helps damp electrostatic waves.

To illustrate the effect of a flat-topped beam profiles, we compare the variation in beam duration $\Delta \tau$ for the three profiles shown in Fig. 4a. In addition to the parabolic beam, there is a purely quartic beam, with no flat region at the beam center, and a beam with the central $60 \%$ of the beam length flat. The plots in Fig. $4 \mathrm{~b}$ of keam duration for fifty laps around the recirculator show that beam shrinkage decreases substantially as the density profile becomes flatter. The reason for this reduced shrinkage is that electric field gradient is confined to a progressively narrow regions at the beam ends as the profile becomes fatter, and inside these regions there is no force to compress the beam. The variation in the average $a$ and $b$ for these cases is effectively the same as in Fig. 1a.

\section{E. Effect of Magnet Errors}

To model the effects of errors in magnet field strengths with CIRCE, the nominal field strength in each magnet may optionally be changed by a randomly chosen amount to give a specified rms error when averaged over the lattice. Errors in quadrupole and bend-magnet strengths are found have quite different effects. Quadrupole errors affect the beam focusing and therefore primarily introduce slice to slice variation in the beam radii, whereas dipole errors effect the beam centroid and cause the centroid to execute a "random walk", with the displacement increasing like the square root of the number of bend magnets.

Error tolerance for field strengths are estimated by rerunning the standard circular lattice with different rnıs errors in the quadrupoles and bends. The error is judged excessive if either the beam radii or the centroid displacement exceed the beam-pipe radius during a fifty lap run. 
We find the beam to be equally sensitive to dipole and quadrupole errors, with rms errors of about $0.01 \%$ or less be required for acceptable transport. Due to the lax criterion used here, this tolerance must be considered to be an upper bound on acceptable errors.

\section{References}

[1] S. S. Yu, J. J. Barnard, G. J. Caporaso, A. Friedman, D. W. Hewitt, H. Kirbie, M. A. Newton, V. K. Neil, A. C. Paul, L. L. Reginato, W. M. Sharp, R. O. Bangerter, C. G. Fong, D. L. Judd, T. F. Godlove, "Constraints on Recirculating Induction-Driver Designs," presented at the 1990 International Symposium of Heavy-Ion Inertial Fusion, Monterey, CA (to be published).

[2] W. M. Sharp, J. J. Barnard, and S. S. Yu, "Envelope Model of a Heavy-Ion Recirculator, ${ }^{\text {, presented at }}$ the 1990 International Symposium of Heavy-Ion Inertial Fusion, Monterey, CA (to be published).

[3] E. P. Lee, E. Close, and L. Smith, "Space Charge Effects in a Bending Magnet System," in Proceeding of the 1987 IEEE Part. Accel. Conf, E. R. Lindstrom and L. S. Taylor, eds. (IEEE, New Jersey, 1987), p. 1126.

[4] L. N. Hand and W. K. H. Panofsky,"Magnetic Quadrupole with Rectangular Aperture," Rev. Sci. Instru., vol. 30, pp. 927-930, October 1959. 